\title{
Series Solution of Non-Similarity Boundary-Layer Flow in Porous Medium
}

\author{
Nabeela Kousar, Rashid Mahmood \\ Department of Mathematics, Faculty of Basic and Applied Sciences, Air University, Islamabad, Pakistan \\ Email: nabeela@mail.au.edu.pk, rashid.mahmood@mail.au.edu.pk
}

Received February 15, 2013; revised March 25, 2013; accepted April 5, 2013

Copyright (C) 2013 Nabeela Kousar, Rashid Mahmood. This is an open access article distributed under the Creative Commons Attribution License, which permits unrestricted use, distribution, and reproduction in any medium, provided the original work is properly cited.

\begin{abstract}
This paper aims to present complete series solution of non-similarity boundary-layer flow of an incompressible viscous fluid over a porous wedge. The corresponding nonlinear partial differential equations are solved analytically by means of the homotopy analysis method (HAM). An auxiliary parameter is introduced to ensure the convergence of solution series. As a result, series solutions valid for all physical parameters in the whole domain are given. Then, the effects of physical parameters $\gamma$ and Prandtl number Pr on the local Nusselt number and momentum thickness are investigated. To the best of our knowledge, it is the first time that the series solutions of this kind of non-similarity boundary-layer flows are reported.
\end{abstract}

Keywords: Non-Similarity; Boundary-Layer Flow; Porous Wedge; Series Solution; Homotopy Analysis Method

\section{Introduction}

Heat transfer continues to be a major field of interest to engineering and scientific researchers, as well as designers, developers, and manufacturers. Heat transfer plays a major role in not only virtually all man made devices, but natural systems as well. Considerable effort has been devoted to research in traditional applications such as chemical processing, general manufacturing, energy devices, including general power systems, heat exchangers, and high performance gas turbines.

The study of boundary-layer flow along surfaces embedded in fluid saturated porous media has received considerable interest, especially in the enhanced recovery of petroleum resources, packed bed reactors and geothermal industries. The fluid flow along a stationary plate is a classical problem of fluid mechanics known as the Blasius problem [1]. In this case the free stream is parallel to the plate and its velocity is constant. If the wall makes a positive angle with the free stream, then the free stream is accelerated along the wall and we have the Falkner? Skan flow along a wedge. Falkner and Skan [2] showed that this problem admits similarity solution as happens with the Blasius problem. Hartree [3] solved this problem and gave numerical results for the wall shear stress for different values of the wedge angle. The heat transfer similarity solution can be developed in the same way by substituting the Falkner-Skan similarity momentum equation into the boundary layer energy equation. Eckert [4] solved the Falkner? Skan flow along an isothermal wedge and gave the first wall heat transfer values. Thereafter, many solutions have been obtained for different aspects of this class of boundary layer problems. Lin and Lin [5] provided very accurate solutions for wall heat transfer from either an isothermal or uniform flux wedge to fluids for any Prandtl number. When the fluid is assumed to have constant properties then the problem is uncoupled, that is, the momentum equation has an influence on the energy equation but the energy equation has not any influence on the momentum equation.

A wide range of applications and a wide variety of analytical and numerical methods have been used to study the heat transport over permeable or impermeable wedge. Elbashbeshy and Dimian [6] investigated the effect of variable viscosity and radiation on flow and heat transfer over a wedge with constant surface temperature but only for $m=1 / 3$ and assuming that the Prandtl number is constant inside the boundary layer. Koh and Hartnett [7] have solved the skin friction and heat transfer for incompressible laminar flow over porous wedges with suction and variable wall temperature. Hsu et al. [8] studied the combined effects of the shape factor, suction/injection rates and viscoelasticity on the flow and temperature 
fields of the flow past a wedge. Magyari and Keller [9] obtained the exact solutions for the two dimensional similarity boundary-layer flows induced by permeable stretching surfaces. Rajagopal et al. [10] gave non-similarity solutions for the flow of a second grade fluid over wedge. Hossain et al. [11] studied the flow of a fluid with temperature dependent viscosity past a permeable wedge with uniform surface heat flux.

From mathematical viewpoints, it is much more difficult to solve a non-linear PDE than ODE. Generally speaking, it is difficult to solve nonlinear PDEs, especially by means of analytic method. Using the perturbation methods or the traditional non-perturbation methods such as Lyapunov's small parameter method [12], the $\delta$-expansion method [13] and Adomian's decomposition method [14], it is difficult to get analytic approximations convergent for all physical parameters in the infinite domain of the flows, because all of these techniques can not ensure the convergence of approximation series. Currently, Cimpean et al. [15] applied the perturbation techniques, combined with numerical techniques, to solve a free convection non-similarity boundary-layer problem over a vertical flat sheet in a porous medium. Like most of perturbation solutions, their results are valid only for small and large $x$, which are regarded as perturbation quantities. Among analytic methods, the method of local similarity is most frequently used. Many researchers [16-19] have obtained the non-similarity solutions by using the method of local similarity. In some cases, the results given by this method agree with numerical solutions. However, the results given by this method are not very accurate and besides are valid only for small $\xi$ in general.

The objective of this paper is to present complete analytic solution to the temperature distribution by means of the homotopy analysis method (HAM). To the best of our knowledge no attempt has been made to present such type of analytic solution to the temperature profile in the non-similarity boundary-layer flows. The HAM aims to solve the various types of nonlinear equations including ordinary differential equations and partial differential equations analytically. Different from perturbation techniques, the HAM is independent of any small/large physical parameters and thus is more general. Besides, it provides us great freedom to choose the initial guess and the auxiliary linear operator so that we can use different types of base functions to approximate the solution. More importantly, the freedom on the choice of the auxiliary parameter provides us a convenient way to ensure the convergence of the solution series. The homotopy analysis method has been successfully applied to complicated nonlinear problems by many researchers [20-29] and so on. In [30] the authors have used the same technique to get the series solutions of of non-similarity boundarylayer flows over the porous wedge. The solutions ob- tained in [30] are uniformly valid for all physical parameters in the whole domain $0 \leq x<\infty$ and $0 \leq y<\infty$. In this paper we have performed heat transfer analysis of steady non-similarity boundary-layer flows over the porous wedge.

\section{Mathematical Formulations}

The equation governing the steady-state, two dimensional, incompressible boundary-layer flow along a porous wedge is given by [30]

$$
f_{\eta \eta \eta}+f f_{\eta \eta}+\beta\left(1-f_{\eta}^{2}\right)=2 \xi\left(f_{\eta} f_{\xi \eta}-f_{\xi} f_{\eta \eta}\right)
$$

subject to the boundary conditions

$$
\begin{aligned}
& \eta=0: f_{\eta}=0, f(\xi, 0)+2 \xi f_{\xi}(\xi, 0)=\gamma \xi^{\kappa} ; \\
& \eta \rightarrow \infty, f_{\eta}=1,
\end{aligned}
$$

where $\gamma=-\sqrt{2} a^{-\kappa-1 / 2} b[v(m+1)]^{\kappa-1 / 2}$, $\kappa=(m+1)^{-1}[n-(m-1) / 2]$, and $\beta=2 m /(m+1)$, are constants. Here, $\gamma$ is the suction/injection parameter, where $b \in R, a>0$ and $v$ is the kinematic viscosity, and $\kappa$ defines the relation between the injection index $n$ and the wedge angle parameter $m$. The dimensionless variables are defined through

$$
\begin{gathered}
\xi=\frac{1}{v} \int_{0}^{x} U(x) \mathrm{d} x=\frac{a x^{m+1}}{v(m+1)}, \\
\eta=\frac{U(x) y}{v \sqrt{2 \xi}}=\sqrt{\frac{a(m+1)}{2 v} x^{\frac{(m-1)}{2}} y,} \\
v=-\sqrt{\frac{a v(m+1)}{2} x^{\frac{m-1}{2}}}\left[f+2 \xi f_{\xi}+\frac{m-1}{m+1} \eta f_{\eta}\right], \\
u=a x^{m} \frac{\partial f}{\partial \eta} .
\end{gathered}
$$

Using the boundary-layer approximations and neglecting the viscous dissipation, the energy equation for temperature $T$ is given by

$$
u \frac{\partial T}{\partial x}+v \frac{\partial T}{\partial y}=\lambda \frac{\partial^{2} T}{\partial y^{2}},
$$

subject to the boundary conditions

$$
T=T_{w} \text {, at } y=0, \text { and } T \rightarrow T_{\infty} \text {, as } y \rightarrow \infty,
$$

where, $\lambda$ is the thermal diffusivity and $T_{w}$ and $T_{\infty}$ are constant temperatures near and far from the surface, respectively.

Introducing the dimensionless temperature $\theta(\xi, \eta)$

$$
\theta(\xi, \eta)=\frac{T_{w}-T}{T_{w}-T_{\infty}},
$$

Equations (3) and (4) readily takes the forms 


$$
\begin{gathered}
\theta_{\eta \eta}+\operatorname{Pr} f \theta_{\eta}=\operatorname{Pr} 2 \xi\left[f_{\eta} \theta_{\xi}-f_{\xi} \theta_{\eta}\right], \\
\theta(0, \xi)=0, \theta(\infty, \xi)=1,
\end{gathered}
$$

where $\operatorname{Pr}=v / \lambda$ is the Prandtl number.

\section{HAM Deformation Equations}

Mathematically, the essence to approximate a nonlinear differential equation is to find a set of proper base functions to fit its solutions. Physically, it is well-known that most viscous flows decay exponentially at infinity (i.e. as $\eta \rightarrow+\infty)$. So, for non-similarity boundary-layer flows, the velocities $u$ and $v$ should decay exponentially at infinity. According to the boundary conditions (2), and (6), $f(\xi, \eta)$ and $\theta(\xi, \eta)$ can be expressed by the set of base function

$$
\left\{\xi^{n} \eta^{k} \mathrm{e}^{-m \lambda \eta} \mid n \geq 0, k \geq 0, m \geq 0, \lambda>0\right\}
$$

in the form

$$
\begin{aligned}
& f(\xi, \eta)=\sum_{m=0}^{\infty} \sum_{n=0}^{\infty} \sum_{k=0}^{\infty} a_{m, n}^{k} \xi^{n} \eta^{k} \mathrm{e}^{-m \lambda \eta}, \\
& \theta(\xi, \eta)=\sum_{m=0}^{\infty} \sum_{n=0}^{\infty} \sum_{k=0}^{\infty} b_{m, n}^{k} \xi^{n} \eta^{k} \mathrm{e}^{-m \lambda \eta}
\end{aligned}
$$

where $a_{m, n}^{k}, b_{m, n}^{k}$ are coefficients and $\lambda>0$ is a scale parameter to be determined. They provide us with the so-called rule of solution expressions for $f(\xi, \eta)$ and $\theta(\xi, \eta)$. According to the rule of solution expressions (7), (8) and from the Equations (2), and (6), it is straight forward to choose the initial approximations

$$
\begin{gathered}
f_{0}(\xi, \eta)=\eta-\frac{\mathrm{e}^{-\lambda \eta}}{\lambda}+\frac{\mathrm{e}^{-2 \lambda \eta}}{\lambda}+\frac{\gamma \xi^{\kappa}}{(1+2 \kappa)} . \\
\theta_{0}(\xi, \eta)=1-\mathrm{e}^{-\lambda \eta},
\end{gathered}
$$

In the frame of HAM, we have great freedom to choose the auxiliary linear operator. However, this freedom is restricted by the solution expression and the boundary conditions, which we must consider in the choice of linear operators. Note that the original governing equations are nonlinear PDEs. In general, PDEs are more difficult to solve than ODEs. So, mathematically, it is much easier to solve a system of nonlinear PDEs if we could choose linear operators which contain derivatives with respect to $\xi$ or $\eta$ only. Physically, for boundary-layer flows, the velocity variation across the flow direction is much larger than in the flow direction. Therefore, the derivatives $\frac{\partial f_{m}}{\partial \eta}, \frac{\partial^{2} f_{m}}{\partial \eta^{2}}, \frac{\partial^{3} f_{m}}{\partial \eta^{3}}$ are considerably larger and thus physically more important than $\frac{\partial f_{m}}{\partial \xi}, \frac{\partial^{2} f_{m}}{\partial \eta \partial \xi}$. Considering all of these, we choose the auxiliary operators

$$
\begin{gathered}
L_{f}=\frac{\partial^{3} f}{\partial \eta^{3}}-\lambda^{2} \frac{\partial f}{\partial \eta}, \\
L_{\theta}=\frac{\partial^{2} \theta}{\partial \eta^{2}}-\lambda^{2} \theta .
\end{gathered}
$$

$L_{f}$ and $L_{\theta}$ have the following properties

$$
\begin{gathered}
L_{f}\left[C_{1}+C_{2} \mathrm{e}^{-\lambda \eta}+C_{3} \mathrm{e}^{\lambda \eta}\right]=0, \\
L_{\theta}\left[C_{4} \mathrm{e}^{-\lambda \eta}+C_{5} \mathrm{e}^{\lambda \eta}\right]=0,
\end{gathered}
$$

where $C_{i}, i=1, \cdots, 5$, are arbitrary constants.

From Equations (1) and (5), we define the nonlinear operators $N_{f}[\Phi(\xi, \eta ; q)]$ and

$$
\begin{aligned}
& N_{\theta}[\Phi(\xi, \eta ; q), \Theta(\xi, \eta ; q)] \\
& N_{f}[\Phi(\xi, \eta ; q)]=\frac{\partial^{3} \Phi(\xi, \eta ; q)}{\partial \eta^{3}}+\Phi \frac{\partial^{2} \Phi(\xi, \eta ; q)}{\partial \eta^{2}} \\
& +\beta\left[1-\left(\frac{\partial \Phi(\xi, \eta ; q)}{\partial \eta}\right)^{2}\right] \\
& -2 \xi\left[\frac{\partial \Phi(\xi, \eta ; q)}{\partial \eta} \frac{\partial^{2} \phi(\xi, \eta ; q)}{\partial \xi \partial \eta}-\frac{\partial \Phi(\xi, \eta ; q)}{\partial \xi} \frac{\partial^{2} \Phi(\xi, \eta ; q)}{\partial \eta \partial \eta}\right], \\
& N_{\theta}[\Phi(\xi, \eta ; q), \Theta(\xi, \eta ; q)] \\
& =2 \xi\left[\frac{\partial \Phi(\xi, \eta ; q)}{\partial \xi} \frac{\partial \Theta(\xi, \eta ; q)}{\partial \eta}-\frac{\partial \Phi(\xi, \eta ; q)}{\partial \eta} \frac{\partial \Theta(\xi, \eta ; q)}{\partial \xi}\right] \\
& +\frac{1}{\operatorname{Pr}} \frac{\partial^{2} \Theta(\xi, \eta ; q)}{\partial \eta^{2}}+\Phi \frac{\partial \Theta(\xi, \eta ; q)}{\partial \eta} .
\end{aligned}
$$

respectively.

Let $c_{0}$ and $c_{1}$ denote the convergence-control parameters [31]. We construct the so-called zeroth-order deformation equations

$$
\begin{aligned}
(1-q) L_{f}\left[\Phi(\xi, \eta ; q)-f_{0}(\xi, \eta)\right]=q c_{0} N_{f}[\Phi(\xi, \eta ; q)] \\
\\
(1-q) L_{\theta}\left[\Theta(\xi, \eta ; q)-\theta_{0}(\xi, \eta)\right] \\
=q c_{1} N_{\theta}[\Phi(\xi, \eta ; q), \Theta(\xi, \eta ; q)],
\end{aligned}
$$

subject to the boundary conditions

$$
\begin{gathered}
\Phi_{\eta}(\xi, 0 ; q)=0, \quad \Theta(\xi, 0 ; q)=0, \\
(1-q)\left[\Phi(\xi, 0 ; q)-f_{0}(\xi, 0)\right] \\
=q c_{2}\left[\Phi(\xi, 0 ; q)+2 \xi \Phi_{\xi}-\gamma \xi^{\kappa}\right], \\
\Phi_{\eta}(\xi, \infty ; q) \rightarrow 1, \quad \Theta(\xi, \infty ; q)=1,
\end{gathered}
$$

where $0 \leq q \leq 1$ is the homotopy-parameter. Clearly, 
when $q=0$ and $q=1$, we have from (17) and (18)

$$
\Phi(\xi, \eta ; 0)=f_{0}(\xi, \eta), \quad \Theta(\xi, \eta ; 0)=\theta_{0}(\xi, \eta),
$$

and

$$
\Phi(\xi, \eta ; 1)=f(\xi, \eta), \quad \Theta(\xi, \eta ; 1)=\theta(\xi, \eta),
$$

Thus, as the homotopy-parameter $q$ increases from 0 to $1, \Phi(\xi, \eta)$ and $\Theta(\xi, \eta)$ move from the initial approximations $f_{0}(\xi, \eta)$ and $\theta_{0}(\xi, \eta)$ to the final solutions $f(\xi, \eta)$ and $\theta(\xi, \eta)$ of Equations (1) and (5), respectively. Assume that the convergence-control parameters are so properly chosen that the Taylor's series of $f(\xi, \eta)$ and $\theta(\xi, \eta)$ expanded with respect to homotopy-parameter $q$, i.e.

$$
\begin{aligned}
& \Phi(\xi, \eta ; q)=\Phi(\xi, \eta ; 0)+\sum_{m=1}^{+\infty} f_{m}(\xi, \eta) q^{m}, \\
& \Theta(\xi, \eta ; q)=\Theta(\xi, \eta ; 0)+\sum_{m=1}^{+\infty} \theta_{m}(\xi, \eta) q^{m},
\end{aligned}
$$

where

$$
\begin{aligned}
& f_{m}(\xi, \eta)=\left.\frac{1}{m !} \frac{\partial^{m} \Phi(\xi, \eta ; q)}{\partial q^{m}}\right|_{q=0}, \\
& \theta_{m}(\xi, \eta)=\left.\frac{1}{m !} \frac{\partial^{m} \Theta(\xi, \eta ; q)}{\partial q^{m}}\right|_{q=0} .
\end{aligned}
$$

converge at $q=1$. Then we have from Equations (22) and (23) that

$$
\begin{gathered}
f(\xi, \eta)=f_{0}(\xi, \eta)+\sum_{m=1}^{+\infty} f_{m}(\xi, \eta), \\
\theta(\xi, \eta)=\theta_{0}(\xi, \eta)+\sum_{m=1}^{+\infty} \theta_{m}(\xi, \eta) .
\end{gathered}
$$

To get the $m$ th-order deformation equations, we first differentiate the zeroth-order deformation Equations (17) and (18) $m$-times with respect to the homotopy-parameter $q$ at $q=0$, and then divide the resulting expression by $m$ !, so that

$$
\begin{aligned}
L_{f}\left[f_{m}(\xi, \eta)-\chi_{m} f_{m-1}(\xi, \eta)\right] & =c_{0} R_{m}\left(\vec{f}_{m-1}\right), \\
L_{\theta}\left[\theta_{m}(\xi, \eta)-\chi_{m} \theta_{m-1}(\xi, \eta)\right] & =c_{1} S_{m}\left(\vec{\theta}_{m-1}\right),
\end{aligned}
$$

subject to the boundary conditions on the wedge

$$
\begin{aligned}
& \theta_{m}(0, \xi)=\left.\frac{\partial f_{m}}{\partial \eta}\right|_{\eta=0}=0, \\
& f_{m}-\chi_{m} f_{m-1}=c_{2}\left[f_{m-1}+2 \xi \frac{\partial f_{m-1}}{\partial \xi}-\gamma \xi^{\kappa}\left(1-\chi_{m}\right)\right], \\
& \text { at } \eta=0,
\end{aligned}
$$

$$
\theta_{m}(\infty, \xi)=\left.\frac{\partial f_{m}}{\partial \eta}\right|_{\eta \rightarrow+\infty}=0
$$

where

$$
\begin{aligned}
& R_{m}\left(\vec{f}_{m-1}\right)=\left.\frac{1}{(m-1) !} \frac{\partial^{m-1} N_{f}[\Phi(\xi, \eta ; q)]}{\partial q^{m-1}}\right|_{q=0}, \\
& S_{m}\left(\vec{\theta}_{m-1}\right)=\left.\frac{1}{(m-1) !} \frac{\partial^{m-1} N_{\theta}[\Theta(\xi, \eta ; q)]}{\partial q^{m-1}}\right|_{q=0},
\end{aligned}
$$

and

$$
\chi_{m}= \begin{cases}0, & m \leq 1, \\ 1, & m>1 .\end{cases}
$$

Now, it is easy to solve the system of linear ordinary differential Equations (28) and (29), subject to the linear boundary conditions (30)-(32). Let $f^{*}(\xi, \eta)$ and $\theta^{*}(\xi, \eta)$ denote the particular solutions of (28)-(29). Using (14), we have the general solutions

$$
\begin{gathered}
f_{m}(\xi, \eta)=f_{m}^{*}(\xi, \eta)+C_{1}+C_{2} \mathrm{e}^{-\lambda \eta}, \\
\theta_{m}(\xi, \eta)=\theta_{m}^{*}(\xi, \eta)+C_{4} \mathrm{e}^{-\lambda \eta},
\end{gathered}
$$

where

$$
\begin{aligned}
& f_{m}^{*}(\xi, \eta)=\chi_{m} f_{m-1}(\xi, \eta)+c_{0} L_{f}^{-1}\left[R_{m}\left(\vec{f}_{m-1}\right)\right], \\
& \theta_{m}^{*}(\xi, \eta)=\chi_{m} \theta_{m-1}(\xi, \eta)+c_{1} L_{\theta}^{-1}\left[S_{m}\left(\vec{\theta}_{m-1}\right)\right],
\end{aligned}
$$

and

$$
\begin{aligned}
C_{1}= & \chi_{m} f_{m-1}(\xi, 0) \\
& +c_{2}\left[f_{m-1}(\xi, 0)+2 \xi \frac{\partial f_{m-1}(\xi, 0)}{\partial \xi}-\gamma \xi^{\kappa}\left(1-\chi_{m}\right)\right](38) \\
& -C_{2}-f_{m}^{*}(\xi, 0) \\
& C_{2}=\left.\frac{1}{\lambda} \frac{\partial f_{m}^{*}}{\partial \eta}\right|_{\eta=0}, \quad C_{4}=-\theta_{m}^{*}(\xi, 0)
\end{aligned}
$$

where $L_{f}^{-1}$ and $L_{\theta}^{-1}$ are inverse operators of $L_{f}$ and $L_{\theta}$, respectively. Therefore, high-order approximations of $f(\xi, \eta)$ and $\theta(\xi, \eta)$ can be obtained, especially by means of symbolic computation.

In many practical applications, the heat transfer rate at the surface, are vital since they influence the quality of the final products. The graphical representation of the results is very useful to discuss the physical features presented by the solutions. The influence of physical parameters on the skin friction coefficient and displacement thisckness are discussed in detail in [30]. Therefore, we confined our attention only on the temperature distribution in the flow field and the effects of physical parame- 
ters on the local Nusselt number and momentum thickness. The local Nusslet number and momentum thickness for non-similarity boundary-layer flows are defined

$$
\begin{gathered}
N u_{x}=-\left.\frac{(m+1) \sqrt{\xi}}{\sqrt{2}} \frac{\partial \theta}{\partial \eta}\right|_{\eta=0}, \\
\delta^{*}(\xi)=\int_{0}^{\infty} f_{\eta}(\xi, \eta)\left[1-f_{\eta}(\xi, \eta)\right] \mathrm{d} \eta .
\end{gathered}
$$

\section{Results and Discussion}

As Liao [32,33] proved in general that, as long as a homotopy-series solution given by the homotopy analysis method is not divergent, it must converge to the exact solution of original nonlinear problems under investigation. The convergence of homotopy-series strongly depend on the convergence-control parameter $c_{0}$. Therefore, the convergence-control parameter provides us a simple way to ensure the convergence of the homotopyseries. Mathematically, the series solutions are dependent upon $c_{0}$. But, physically, the solution is independent of the convergence-control parameters. As a result, the homotopy-series must converge to the same result for all corresponding values of $c_{0}$ which ensures the convergence. As mentioned by Liao [32], the admissible values of $c_{0}$ for which the homotopy-series converges can be determined by plotting the so-called $c_{0}$-curves or by plotting the residual error verses $c_{0}$. Besides, the optimal homotopy-analysis approach [31] can be used to find the optimal convergence-control parameters. The problem under consideration was solved by Kousar and Liao [30] for velocity field over permeable wedge. In [30] the authors have shown that the homotopy-series (24) converges at $c_{0}=-1$ for all physical parameters in the whole domain $0 \leq x<\infty$ and $0 \leq y<\infty$. Therefore, in the present analysis, we need only to investigate the convergence of the series (25). In order to make series (25) convergent, let $\delta_{m}(\xi, \eta)$ denotes the residual error of the $m$ th-order homotopy-series (25), and

$E(h)=\Delta=\iint \delta_{m}^{2}(\xi, \eta) \mathrm{d} \xi \mathrm{d} \eta$ denotes the integral of the residual error. Plotting the curves of $\Delta \sim c_{0}$, it is straight forward to find a region of $c_{0}$ in which $\Delta$ decreases to zero as the order of approximation increases. In this way, we can get the best value of $c_{0}$ corresponding to the minimum of the residual error of the original governing equation.

For example, the approximate region for the convergence of the homotopy-series is about $-3 / 2 \leq c_{0} \leq 0$ as shown in Figure 1 for different values of the Prandtl numbers when given $\lambda=5, \beta=1, \kappa=1 / 2$ and $\gamma=1$.

In general, we can substitute the series solutions for the governing equations and evaluate the square residual error so as to check the convergence of the solutions.

Table 1 shows the square residual error of (5). It is

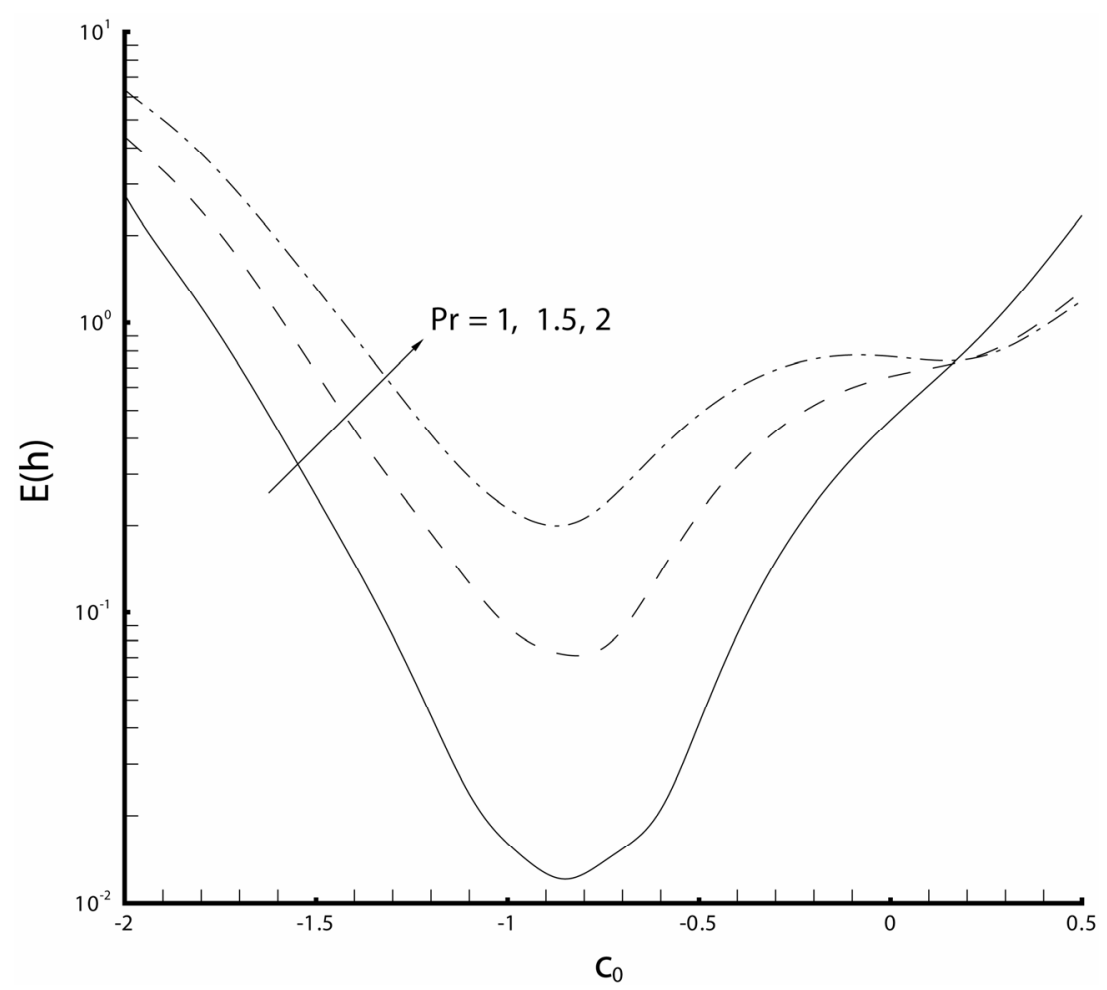

Figure 1. The 8th-order approximations of square residual error versus convergence-control parameter for different Prandtl numbers when $\beta=1, \kappa=1 / 2, \gamma=1$ by mean of $\lambda=5$. 
seen that by increasing the order of approximation the square residual error decreases. This indicates that our HAM series solution is convergent. Similarly, in case of $\gamma=-1 / 4$ with $\beta=1, \operatorname{Pr}=1$ and $\kappa=1 / 2$, our series solution converges by means of $\lambda=5$ and $c_{0}=-1$. It is found that, in general, our series solutions converge by means of $\lambda=5$ and $c_{0}=-1$ in the whole spatial domain. Besides, the so-called homotopy-Padé technique [32] is used to accelerate the convergence of the homotopy-series solution.

Figures 2 and 3 represent the effect of the parameter $\gamma$ on the local Nusslet number and momentum thickness for $\beta=1, \kappa=1 / 2, \operatorname{Pr}=1$ by means of $\lambda=5$ and $c_{0}=c_{1}=-1$. It is clear from the figures that the injection increases the momentum thickness and decreases the heat transfer rate at surface, but the suction decreases thickness and increases the heat transfer rate. This is due to the fact that the shear stress increases when introducing suction, which in turn increases the local Nusselt number.

Table 1. Square residual error when $\beta=1, \gamma=1, \operatorname{Pr}=1$ and $\kappa=1 / 2$ by means of $\lambda=5, c_{0}=-1$ and $c_{1}=-1$.

\begin{tabular}{cc}
\hline Order of approximation & Residual error \\
\hline 1 st & 0.321604 \\
5 th & 0.040759 \\
10 th & 0.010320 \\
15 th & 0.005176 \\
\hline
\end{tabular}

The heat transfer parameter increases significantly with the Prandtl number, as highjer Prandtl number has lower thermal conductivity, which results in thinner thermal boundary-layer and hence, higher heat transfer rate at the surface. It is also noted that the non-similarity solutions are very close to the similarity ones as $\xi \rightarrow 0$ for $\kappa>0$.

Figures 4 and 5 show the influence of $\gamma$ on the momentum thickness and the local Nusselt number when $\kappa=-1 / 4, \beta=1, \operatorname{Pr}=1$ by means of $\lambda=5$ and $c_{0}=c_{1}=$ -1 . Again, it is observed from these figures that the suction decreases the thickness but increases the local Nusselt number while the injection increases the thickness but decreases the local Nusselt number. It is also observed that the non-similarity flows are close to the similarity ones as $\xi \rightarrow \infty$. Hence, the non-similarity flows in the region $\xi \rightarrow 0$ for $\kappa>0$ and $\xi \rightarrow \infty$ for $\kappa<0$ are very close to the similarity ones, respectively.

Figure 6 shows the heat transfer parameter for various values of Prandtl numbers (Pr) when the other physical parameters are fixed. It is observed that heat transfer parameter increases as Pr increases. This is because the fluid with higher Prandtl number has a relatively low thermal conductivity, which reduces the conduction and thereby reduces the thermal boundary-layer thickness. As a consequence the heat transfer rate at the surface increases.

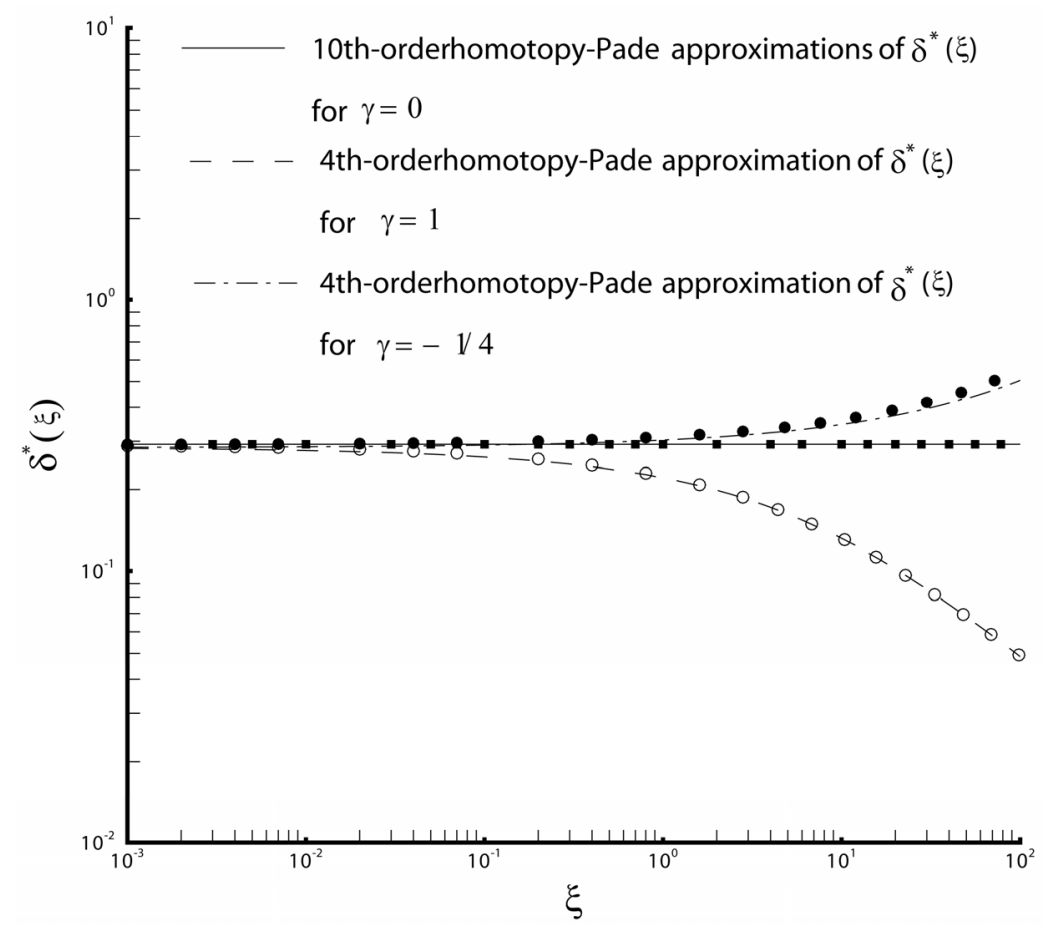

Figure 2. Influence of $\gamma$ on the momentum thickness $\delta^{*}(\xi)$ when $\beta=1, \kappa=1 / 2, \operatorname{Pr}=1$ by means of $c_{0}=c_{1}=-1$ and $\lambda$ = 5. Squares: $[12,12]$ homotopy-Padé approximation for $\gamma=0$; Open circles: $[8,8]$ homotopy-Padé approximation for $\gamma=1$, Filled circles: $[8,8]$ homotopy-Padé approximation for $\gamma=-1 / 4$. 


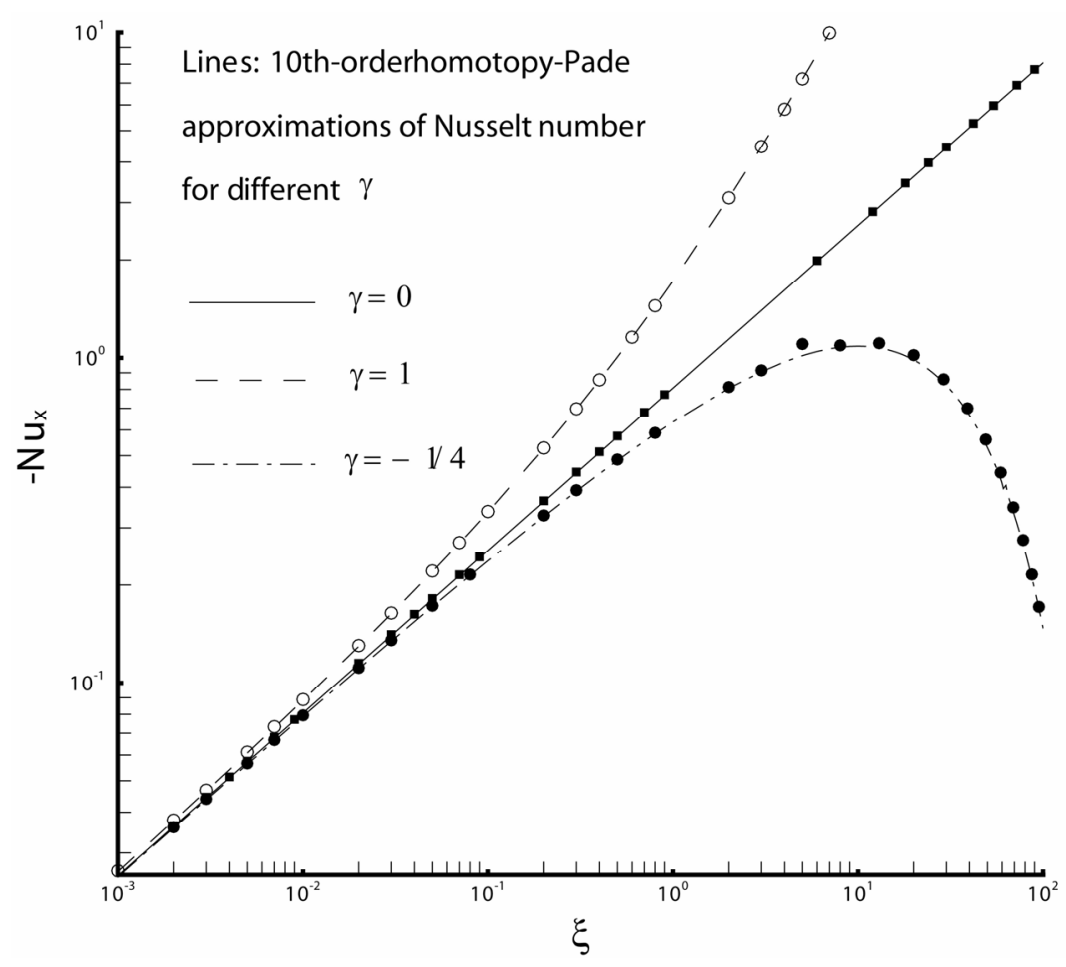

Figure 3. The 8th-order homotopy-Padé approximation of the local Nusselt number $\mathrm{Nu}_{x}$ for different $\gamma$ when $\beta=1$, $\kappa=1 / 2, \operatorname{Pr}=1$ by means of $c_{0}=c_{1}=-1$ and $\lambda=5$. Squares: $[12,12]$ homotopy-Padé approximation for $\gamma=0$; Open circles: $[8,8]$ homotopy-Padé approximation for $\gamma=1$; Filled circles: $[8,8]$ homotopy-Padé approximation for $\gamma=-1 / 4$.

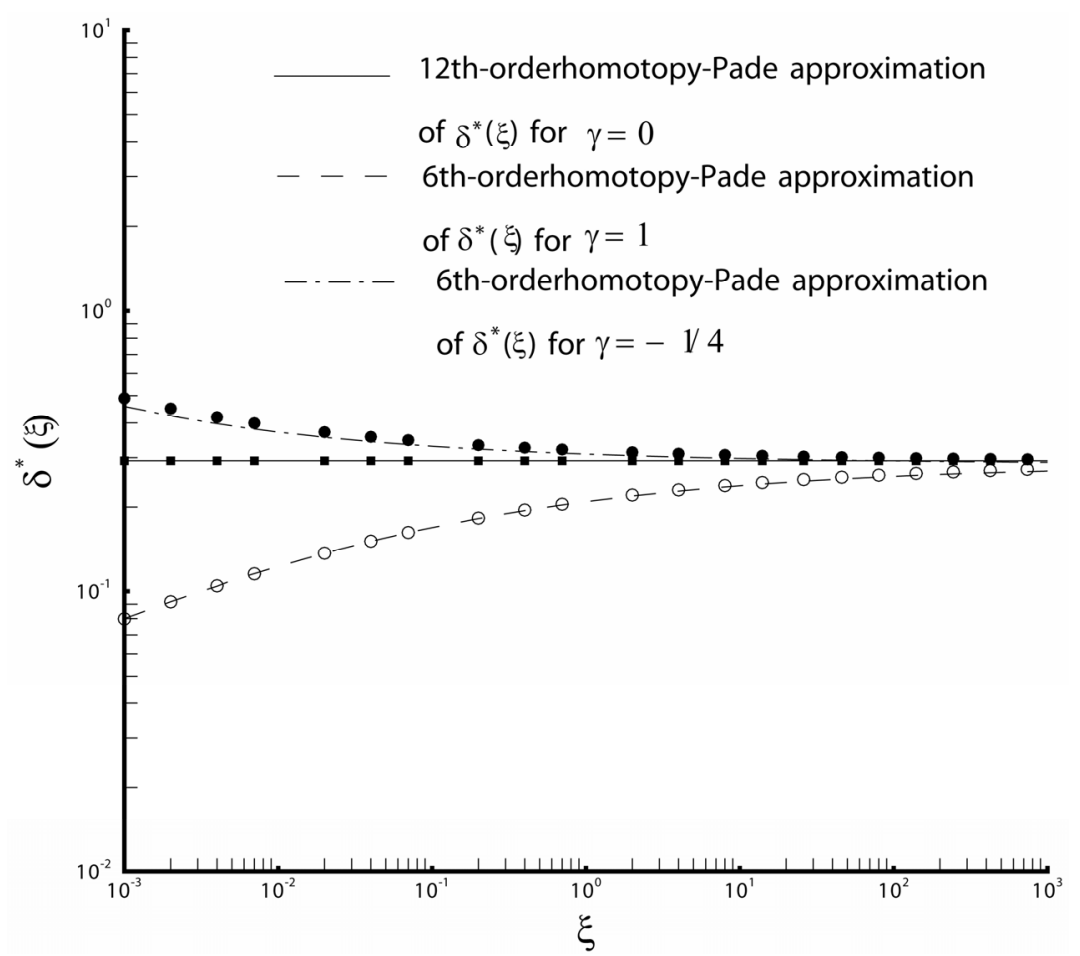

Figure 4. Influence of $\gamma$ on the momentum thickness $\delta^{*}(\xi)$ when $\beta=1, \kappa=-1 / 4, \operatorname{Pr}=1$ by means of $c_{0}=c_{1}=-1$ and $\lambda=5$. Squares: $[10,10]$ homotopy-Padé approximation for $\gamma=0$; Open circles: $[8,8]$ homotopy-Padé approximation for $\gamma=1$; Filled circles: $[8,8]$ homotopy-Padé approximation for $\gamma=-1 / 4$. 


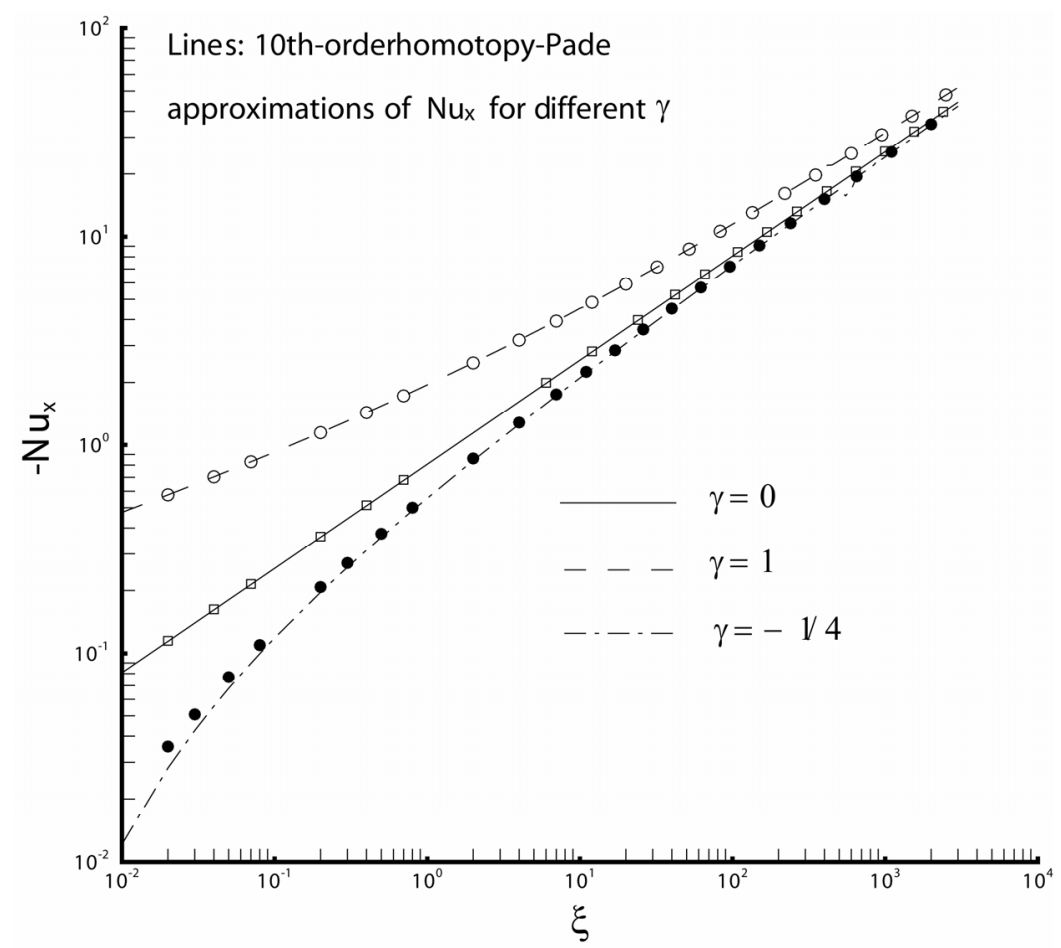

Figure 5. The 8th-order homotopy-Padé approximation of the local Nusselt number $\mathbf{N u}_{x}$ for different $\gamma$ when $\beta=1$, $\kappa=-1 / 4, \operatorname{Pr}=1$ by means of $c_{0}=c_{1}=-1$ and $\lambda=5$. Squares: $[12,12]$ homotopy-Padé approximation for $\gamma=0$; Open circles: [6,6] homotopy-Padé approximation for $\gamma=1$; Filled circles: $[6,6]$ homotopy-Padé approximation for $\gamma=-1 / 4$.

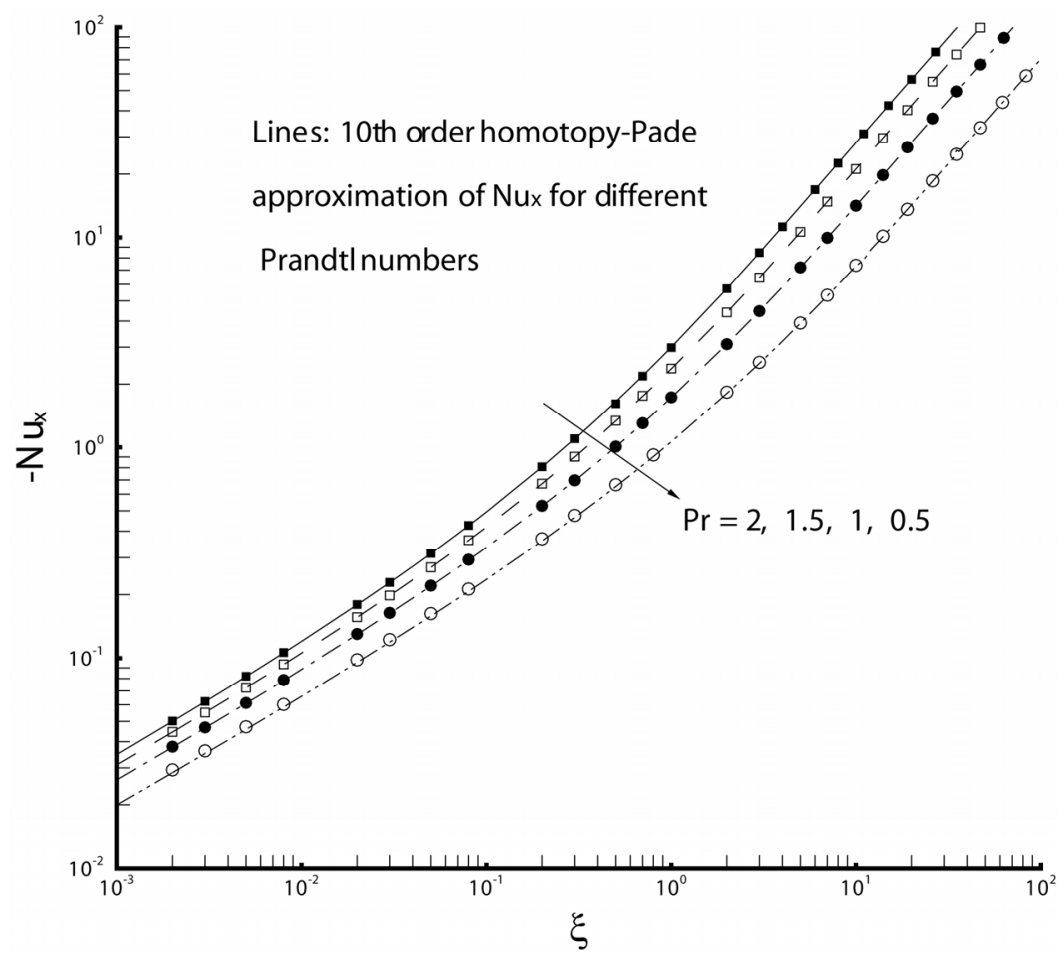

Figure 6. The 8th-order homotopy-Padé approximation of the local Nusselt number $\mathrm{Nu}_{x}$ for different $\operatorname{Pr}$ when $\beta=1$, $\gamma=1, \kappa=1 / 2$ by means of $\lambda=5$. Squares: $\operatorname{Pr}=2$ by means of $c_{0}=c_{1}=-1$; Open squares: $\operatorname{Pr}=1.5$ by means of $c_{0}=c_{1}=-1$; Circles: $\operatorname{Pr}=1$ by means of $c_{0}=c_{1}=-1$; Filled circles: $\operatorname{Pr}=0.5$ by means of $c_{0}=-1$ and $c_{1}=-1 / 2$. 


\section{Conclusion}

In this paper, the non-similarity boundary-layer flows with heat transfer analysis is studied. Complete analytic solutions which are uniformly valid for all the physical parameters in the whole spatial region are obtained by an analytic technique for strongly nonlinear problems, namely the homotopy analysis method. Besides, the so-called homotopy-Padétechnique is applied to accelerate the convergence of the homotopy-series solutions. Then, the effects of the physical parameters on local Nusselt number and momentum thickness are investigated. To the best of our knowledge, it is the first time that the series solutions of this kind of non-similarity boundary-layer flows for temperature distribution are reported. This analytic approach has general meanings and can be used to solve other non-similarity boundary-layer flows in a similar way.

\section{Acknowledgements}

The author wish to particularly thank Prof. Shijun Liao for his valuable suggestions and guidance.

\section{REFERENCES}

[1] H. Blasius, "Grenzschichten in Füssigketiten mit Kleiner Reibung," Zeitschrift für Angewandte Mathematik und Physik, Vol. 56, 1908, pp. 1-37.

[2] V. M. Falkner and S. W. Skan, "Some Approximate Solutions of the Boundary-Layer Equations," Philosophical Magazine, Vol. 12, 1931, pp. 865-896.

[3] D. R. Hartree, "On an Equation Occurring in Falkner and Skan's Approximate Treatment of the Equations of the Boundary Layer," Proceedings of the Cambridge Philosophical Society, Vol. 33, No. 2, 1937, pp. 223-239. doi:10.1017/S0305004100019575

[4] E. R. G. Eckert, "Die Berechnung des Warmeuberganges in der Laminaren Grenzschicht um Stromter Korper," VDI-Forschungsheft, Vol. 416, 1942, pp. 1-24.

[5] H.-T. Lin and L.-K. Lin, "Similarity Solutions for Laminar Forced Convection Heat Transfer from Wedges to Fluids of Any Prandtl Number," International Journal of Heat and Mass Transfer, Vol. 30, No. 6, 1987, pp. 11111118. doi:10.1016/0017-9310(87)90041-X

[6] E. M. A. Elbashbeshy and M. F. Dimian, "Effect of Radiation on the Flow and Heat Transfer over a Wedge with Variable Viscosity," Applied Mathematics and Computation, Vol. 132, No. 2-3, 2002, pp. 445-454. doi:10.1016/S0096-3003(01)00205-3

[7] J. C. Y. Koh and J. P. Hartnett, "Skin-Friction and Heat Transfer for Incompressible Laminar Flow over Porous Wedges with Suction and Variable Wall Temperature," International Journal of Heat and Mass Transfer, Vol. 2, No. 3, 1961, pp. 185-198. doi:10.1016/0017-9310(61)90088-6

[8] C. H. Hsu, C. H. Chen and J. T. Teng, "Temprature and
Flow Fields for the Flow of a Second Grade Fluid Past a Wedge," International Journal of Non-Linear Mechanics, Vol. 32, No. 5, 1997, pp. 933-946.

[9] E. Magyari and B. Keller, "Exact Solutions for Self-Similar Boundary Layer Flows Induced by Permeable Stretching Walls," European Journal of Mechanics: B/Fluids, Vol. 19, No. 1, 2000, pp. 109-122. doi:10.1016/S0997-7546(00)00104-7

[10] K. R. Rajagopal and A. S. Gupta, "An Exact Solution for the Flow of a Non-Newtonian Fluid Past an Infinite Porous Plate," Meccanica, Vol. 19, No. 2, 1984, pp. 158160. doi:10.1007/BF01560464

[11] M. A. Hossain, M. Z. Munir, M. S. Hafiz and H. S. Takhar, "Flow of Viscous Incompressible Fluid with Temperature Dependent Viscosity Past a Permeable Wedge with Uniform Surface Heat Flux," Heat and Mass Transfer, Vol. 36, No. 4, 2000, pp. 333-341. doi:10.1007/s002310000079

[12] A. M. Lyapunov, "General Problem on Stability of Motion (English Translation)," Taylor and Francis, London, 1992.

[13] A. V. Karmishin, A. I. Zhukov and V. G. Kolosov, "Methods of Dynamics Calculation and Testing for ThinWalled Structures," Mashinostroyenie, Moscow, 1990.

[14] G. Adomian, "Solving Frontier Problems of Physics: The Decomposition Method," Kluwer Academic Publishers, Boston, 1994. doi:10.1007/978-94-015-8289-6

[15] D. Cimpean, J. H. Merkin and D. B. Ingham, "On a Free Convection Problem over a Vertical Flat Surface in a Porous Medium," Transport Porous, Vol. 64, No. 3, 2006, pp. 393-411. doi:10.1007/s11242-005-5236-y

[16] M. Massoudi, "Local Non-Similarity Solutions for the Flow of a Non-Newtonian Fluid over a Wedge," International Journal of Non-Linear Mechanics, Vol. 36, No. 6, 2001, pp. 961-976. doi:10.1016/S0020-7462(00)00061-5

[17] K. J. Wanous and E. M. Sparrow, "Heat Transfer for Flow Longitudinal to a Cylinder with Surface Mass Transfer," Journal of Heat Transfer, Vol. 87, No. 2, 1965, pp. 317 319. doi:10.1115/1.3689103

[18] D. Catherall, K. Stewartson and P. G. Williams, "Viscous Flow Past a Flat Plate with Uniform Injection," Proceedings of the Royal Society A, Vol. 284, No. 1398, 1965, pp. 370-396. doi:10.1098/rspa.1965.0069

[19] E. M. Sparrow and H. S. Yu, "Local Non-Similarity Thermal Boundary-Layer Solutions," Journal of Heat Transfer: Transactions of the ASME, Vol. 93, No. 4, 1971, pp. 328-334.

[20] C. Wang, S. J. Liao and J. M. Zhu, “An Explicit Analytic Solution for Non-Darcy Natural Convection over Horizontal Plate with Surface Mass Flux and Thermal Dispersion Effects," Acta Mechanica, Vol. 165, No. 3-4, 2003 , pp. 139-150. doi:10.1007/s00707-003-0039-0

[21] T. Hayat, M. Khan and M. Ayub, "On the Explicit Analytic Solutions of an Oldroyd 6-Constant Fluid," International Journal of Engineering Science, Vol. 42, No. 2, 2004, pp. 123-135. doi:10.1016/S0020-7225(03)00281-7

[22] S. J. Liao, "A New Branch of Solutions of BoundaryLayer Flows over an Impermeable Stretched Plate," In- 
ternational Journal of Heat and Mass Transfer, Vol. 48, No. 12, 2005, pp. 2529-2539.

doi:10.1016/j.ijheatmasstransfer.2005.01.005

[23] S. J. Liao and E. Magyari, "Exponentially Decaying Boundary Layers as Limiting Cases of Families of Algebraically Decaying Ones," Zeitschrift für Angewandte Mathematik und Physik, Vol. 57, No. 5, 2006, pp. 777-792. doi:10.1007/s00033-006-0061-x

[24] S. P. Zhu, "A Closed-Form Analytical Solution for the Valuation of Convertible Bonds with Constant Dividend Yield," ANZIAM Journal, Vol. 47, No. 4, 2006, pp. 477494. doi:10.1017/S1446181100010087

[25] S. P. Zhu, "An Exact and Explicit Solution for the Valuation of American Put Options," Quantitative Finance, Vol. 6, No. 3, 2006, pp. 229-242. doi:10.1080/14697680600699811

[26] M. Yamashita, K. Yabushita and K. Tsuboi, "An Analytic Solution of Projectile Motion with the Quadratic Resistance Law Using the Homotopy Analysis Method," Journal of Physics A, Vol. 40, No. 29, 2007, pp. 8403-8416. doi:10.1088/1751-8113/40/29/015

[27] H. Song and L. Tao, "Homotopy Analysis of 1D Unsteady, Nonlinear Groundwater Flow through Porous Media,"
Journal of Coastal Research, Vol. 50, 2008, pp. 292-295.

[28] W. H. Cai, "Nonlinear Dynamics of Thermal-Hyraulic Networks," Ph.D. Thesis, University of Notre Dame, 2006.

[29] H. Xu and I. Pop, "Homotopy Analysis of Unsteady Boundary-Layer Flow Started Impulsivley from Rest along a Symmetric Wedge," Journal of Applied Mathematics and Mechanics, Vol. 88, No. 6, 2008, pp. 507-514.

[30] N. Kousar and S. J. Liao, "Series Solution of Non-Similarity Boundary-Layer Flows over a Porous Wedge," Transport in Porous Media, Vol. 83, No. 2, 2010, pp. 397-412.

[31] S. J. Liao, "An Optimal Homotopy-Analysis Approach for Strongly Nonlinear Differential Equations," Coтmunications in Nonlinear Science and Numerical Simulation, Vol. 15, No. 8, 2010, pp. 2003-2016.

[32] S. J. Liao, "Beyond Perturbation: Introduction to the Homotopy Analysis Method," Chapman and Hall/CRC Press, Boca Raton, 2003. doi:10.1201/9780203491164

[33] S. J. Liao, "The Proposed Homotopy Analysis Technique for the Solution of Nonlinear Problems," Ph.D. Thesis, Shanghai Jiao Tong University, Shanghai, 1992. 